\title{
Effect of the Management Tool for Daily Life Performance on Patients with Cardiovascular Disease: A Randomized Controlled Trial
}

\author{
Megumi Fukui, MS, OTR ${ }^{\text {a,b }}$ Yosuke Yoshida, PhD, RPT a and Kazuo Higaki, PhD, OTR ${ }^{\text {c }}$
}

\begin{abstract}
Objective: Cardiovascular diseases cause psychological symptoms, such as depression and anxiety. Symptoms of depression have a major effect on patients and worsen prognosis as a result of reduced quality of life and decreased levels of physical activity. Because cardiac rehabilitation (CR) has physical, mental, and secondary preventative effects, it is necessary to evaluate the psychological factors of patients and to provide patients with psychological support based on the results. The management tool for daily life performance (MTDLP) was developed in 2006 by the Japanese Association of Occupational Therapists. Since then, its effectiveness for patients with cerebrovascular diseases and bone fractures has been verified. However, no randomized controlled trial has been conducted on the effectiveness of interventions using MTDLP on patients with cardiovascular diseases. Methods: We examined the effectiveness of intervention using MTDLP on patients undergoing outpatient CR. Thirty-six patients who scored at least 48 on the self-rating depression scale (SDS) were included in the study. Eighteen patients were allocated to both the CR and MTDLP groups. The SDS, Barthel index, Frenchay Activities Index (FAI), and Life-Space Assessment were evaluated as outcome measures. Results: The CR group $(\mathrm{n}=14)$ showed significantly improved post-intervention scores on the SDS ( $\mathrm{P}=0.007)$. Furthermore, the MTDLP group $(\mathrm{n}=11)$ showed significantly improved post-intervention scores on the SDS $(\mathrm{P}=0.010)$ and FAI $(\mathrm{P}=0.003)$. Conclusions: $\mathrm{CR}$ improves depression, whereas additional intervention using MTDLP improves not only depression but also various daily activities. Consequently, intervention using MTDLP in CR appears to be effective.
\end{abstract}

Key Words: activities parallel to daily living; cardiovascular diseases; depression; management tool for daily life performance

\section{INTRODUCTION}

Cardiovascular diseases engender psychological symptoms, such as anxiety and depression. Patients with myocardial infarction are 3 times more likely than healthy individuals to suffer from depression. ${ }^{1)}$ The prevalence of depression in patients with heart failure is $22 \%,{ }^{2}$ ) and patients with congestive heart failure are 1.8 times more likely to die if they experience depression comorbidity. ${ }^{3)}$ It has been reported that the prevalence of depression in Japanese adults is approximately $\left.3 \%,{ }^{4}\right)$ indicating that the prevalence of depression in patients with heart failure is much higher than that in the general population. Furthermore, the incidence of depression reportedly increases as the severity of heart failure increases. ${ }^{2}$ ) The symptoms of depression greatly affect patients and worsen prognosis because of reduced quality of life (QOL), poor medication adherence, and reduced levels of physical activity. ${ }^{5-7)}$ Drug therapy and psychotherapy form the basis of therapeutic strategies for the symptoms of depression. Thombs et al. ${ }^{8)}$ reported that the symptoms of depression improve when drug therapy alone is used; however, this approach did not improve cardiac disease recurrence or

Received: July 25, 2018, Accepted: January 17, 2019, Published online: February 5, 2019

${ }^{\text {a }}$ Department of Rehabilitation, Nara Prefectural Seiwa Medical Center, Nara, Japan

${ }^{\mathrm{b}}$ Research Institute of Rehabilitation Sciences, Osaka Prefectural University, Osaka, Japan

c Department of Community Health, Osaka Prefectural University, Osaka, Japan

Correspondence: Megumi Fukui, MS, OTR, Department of Rehabilitation, Nara Prefectural Seiwa Medical Center, 1-14-16 Mimuro,

Sango-cho, Ikoma-gun, Nara 636-0802, Japan, E-mail: fukuimegumiot@gmail.com

Copyright (C) 2019 The Japanese Association of Rehabilitation Medicine 
mortality rate.

Exercise therapy plays a central role in cardiac rehabilitation (CR) and is physically and psychologically effective; moreover, it is effective in secondary prevention and improving QOL. ${ }^{5)}$ Additionally, exercise therapy has been proven effective in improving depression ${ }^{7,9,10)}$; consequently, it is necessary to evaluate the psychological factors of patients and to deliver psychological support when providing them with exercise therapy. ${ }^{11)}$

Since 2008, to maintain and improve the health of the elderly, the Japanese Association of Occupational Therapists (JAOT), under the auspices of the Ministry of Health, Labor and Welfare of Japan, developed a management tool that contributes to comprehensive community care and that is easily understandable by the general public. This tool is known as the management tool for daily life performance (MTDLP). It is a comprehensive management tool which facilitates the support of patient independence. Its effect has been verified in various clinical settings, including elderly day care services, institutionalized services, and medical care. Interventions using MTDLP have been shown to improve activities of daily living (ADL), the level of independence of activities parallel to daily living (APDL), performance of social roles, and health-related QOL. ${ }^{12,13)}$ Although its effectiveness for most patients with cerebrovascular diseases and bone fractures has been verified, no randomized controlled trial concerning the effectiveness of interventions using MTDLP for patients with cardiovascular diseases has yet been performed.

Duruturk et al. ${ }^{14)}$ reported that patients with cardiac diseases face difficulty with a wide range of life activities, such as walking, climbing stairs, bathing, dressing, and outings. Intervention using MTDLP was expected to improve items associated with ADL, APDL, and the performance of social roles when used for patients with cardiac diseases. The present study aimed to examine the effectiveness of interventions using MTDLP on patients with cardiovascular diseases.

\section{METHODS}

\section{Study Design}

This study was a randomized controlled trial that compared the effects of intervention using MTDLP on one group that underwent $\mathrm{CR}$ only (CR group) and another group that underwent intervention using MTDLP in addition to CR (MTDLP group). The allocation of subjects to groups was done by a third party. Further, this study was a single-blind study, wherein the patients were unaware of the group to which they had been assigned.

\section{Subjects}

Patient characteristics such as sex; age; body mass index (BMI); primary disease; cardiac function [left ventricular ejection fraction (LVEF), the ratio of early mitral inflow velocity and mitral annular early diastolic velocity (E/e'), and the New York Heart Association (NYHA) classification of cardiac performance]; renal function (eGFR); and exercise tolerance functions [grip strength, knee extension strength, and the Timed Up and Go (TUG) test] were obtained from previous medical records.

\section{Interventions}

At the Nara Prefectural Seiwa Medical Center, outpatient $\mathrm{CR}$ lasts approximately $60 \mathrm{~min}$ per session. According to the treatment guidelines for circulatory diseases in Japan, ${ }^{4)}$ after having their blood pressure and body weight measured, the patients perform exercises using an ergometer set at between 11 and 13 on the Borg scale, undergo resistance training using an exercise band (Can do AccuForce Corp.), and finally perform cool-down exercises. Both the CR and the MTDLP groups participated in outpatient CR at separately determined frequencies. The frequency was once a week for 150 days from the start of CR.

In the MTDLP group, an occupational therapist (OT) conducted an intervention using MTDLP in addition to normal CR. At the initial interview, lasting approximately 20-30 min, the OT used the MTDLP sheet and asked each patient about the life activities that they wanted to improve. ${ }^{15)}$ Furthermore, the OT predicted the possibility of achievement and the level for the goal and set up a specific support plan. This allowed the OT to set goals that could reasonably be expected to lead to behavior modification. To avoid overloading, these goals were set in consideration of each patient's cardiac function and Borg scale scores for exercise while undergoing CR. The goals and plans to achieve them were shared with the entire CR staff, and the interventions were performed by OT and CR staff. The interventions were conducted by three OTs with basic training in MTDLP provided by the JAOT. To eliminate differences in terms of OT intervention, the OT conducted intervention using MTDLP after discussion with the other OTs.

\section{Outcome Measures}

Four items that were included in the self-administered questionnaires were evaluated. The questionnaires were completed by patients in both groups before and after the 
3-month intervention period. The self-rating depression scale (SDS) score, which assesses the level of depression, was used as the primary outcome measure. The total SDS score is the sum of each score of 20 items associated with the patient's depressive mood. The score ranges from 20 to 80 , and scores $>40$ reflect a depressive mental state. The score for each item is in the range $1-4 .{ }^{16)}$ In the current study, the cutoff score was $48,{ }^{17,18)}$ and a score of $\geq 48$ indicated depression. ADL was assessed using the Barthel index (BI), ${ }^{19)}$ APDL was assessed using the Frenchay Activities Index (FAI), ${ }^{20,21)}$ and life space was assessed using the Life-Space Assessment (LSA).

The BI, a scale measuring disability or dependence in ADL, was originally developed to measure ADL in stroke patients; it was subsequently extended to geriatric patients. ${ }^{22)}$ The score ranges from 0 to 100 . Although not all patients with low ADL are frail, a low BI score is generally associated with high levels of frailty. ${ }^{23)}$ The $\mathrm{BI}$ is a 10 -item scale that assesses a patient's ability to feed, groom, bathe, use the toilet, dress, walk, transfer, and climb stairs, and also includes fecal incontinence and urinary incontinence. The FAI includes 15 items covering various applied activities in subjects' daily and social lives, e.g., shopping, meal preparation, walking outdoors, and hobbies. The score ranges from 0 to 45 . It scores each item from 0 to 3 based on the frequency of the activity over a 3- or 6-month period. The FAI assesses activity frequency and provides a clearer overview of subjects' living situations. Furthermore, scoring is simple, and the reliability and validity of the FAI as an evaluation method have been confirmed in Japan. ${ }^{24)}$ Recent studies using the LSA, as proposed by Baker et al., ${ }^{25)}$ have reported activity levels among community-dwelling elderly individuals, and the scale's reliability and validity have been verified. ${ }^{25,26)}$ The LSA is a useful scale for quantitatively assessing the level, frequency, and range of activities of elderly individuals and their degree of dependence. The score ranges from 0 (mobility confined to one's bedroom) to 120 (traveled out of town every day without assistance from another person or an assistive device). ${ }^{25)}$

\section{Data Analysis}

The Shapiro-Wilk test was used to assess the normality of data. Inter-group comparisons of BMI, LVEF, eGFR, grip strength, knee extension strength, and TUG test results were conducted using unpaired $t$-tests. Inter-group comparisons of age and the E/e' ratio were conducted using the MannWhitney $U$-test, and inter-group sex-based comparisons and NYHA classification comparisons of cardiac performance were conducted using Fisher's exact test. Inter-group comparisons of primary diseases were conducted using the chisquare test of independence.

The efficacy of intervention was assessed by comparing the SDS, BI, FAI, and LSA scores before and after intervention using the Wilcoxon signed-rank test. We also calculated the effect size, which was denoted by Pearson's correlation coefficient. The effect sizes were interpreted as small $(\mathrm{r}=0.10)$, medium $(\mathrm{r}=0.30)$, or large $(\mathrm{r}=0.50)$. In addition, we derived the main effect and interaction using two-way analysis of variance. Statistical analyses were performed using SPSS ver. 25 , and $\mathrm{P}<0.05$ was considered statistically significant.

\section{Ethical Considerations}

Oral and written descriptions of the objective of the study were provided to the patients, and they provided written consent prior to beginning the study. Furthermore, they voluntarily participated and were free to withdraw from the study at any time. The study followed the ethical standards of the Declaration of Helsinki and was approved by the Institutional Review Boards of the Nara Prefecture Seiwa Medical Center (no. 67) and the Department of Research, Institute of Rehabilitation Sciences, Osaka Prefecture University (\#2016-215). This trial was registered with the University Hospital Medical Information Network Clinical Trial Registry (UMIN-CTR 000033516, Registered date: Jul 25, 2018, https://upload.umin.ac.jp/cgi-bin/ctr_e/ctr_view. cgi?recptno=R000038217).

\section{RESULTS}

\section{Study Protocol}

The questionnaire survey was conducted between August and September 2017. In total, 36 patients (25 men, 11 women; mean age, $75.9 \pm 6.8$ years) were selected from among 122 patients who underwent outpatient CR at the Nara Prefectural Seiwa Medical Center. Thirty six patients who scored at least 48 on the SDS were included. Eighteen patients each were allocated to the CR and MTDLP groups.

The intervention period was 3 months (November 2017 January 2018). Four patients in the CR group dropped out during the 3-month intervention period because of completion of their CR program or hospitalization that resulted from cardiac disease progression. Seven patients in the MTDLP group dropped out during the 3-month intervention period because of completion or discontinuation of their CR program or hospitalization resulting from cardiac disease progression or compression fracture. Consequently, 14 and 


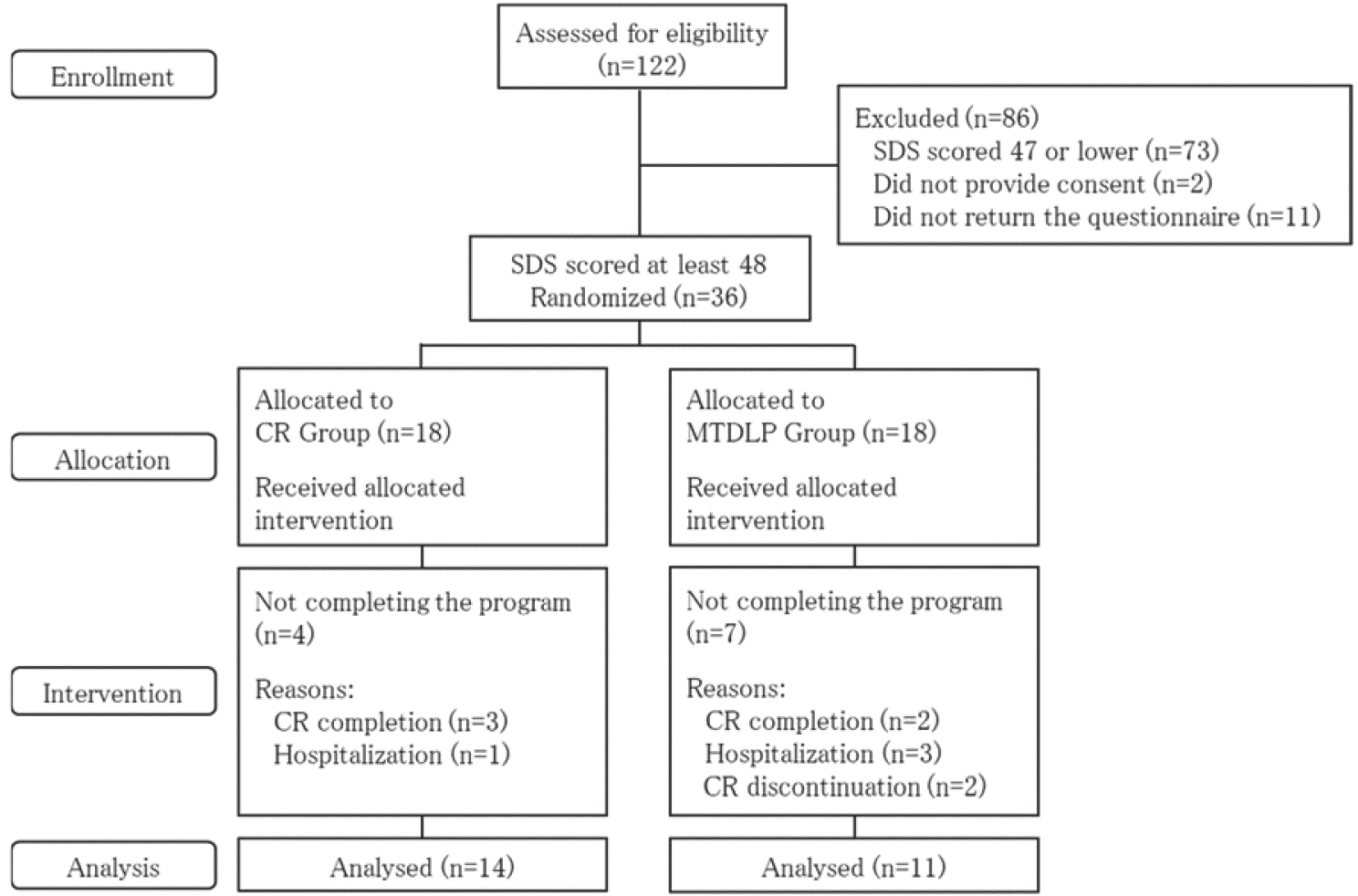

Fig. 1. CONSORT flowchart showing the flow of patients through the trial.

11 patients in the CR and MTDLP groups, respectively, were included for per protocol set analysis (Fig. 1). No changes in the daily life environment or other factors related to the analysis population were observed during the intervention period.

\section{Comparison of Patient Characteristics}

The basic characteristics of the patients in both groups used in our baseline analysis are presented in Table 1. No significant difference was observed for any item.

\section{Comparison of Outcome Measures Before and after Intervention}

Changes in pre- and post-intervention scores are presented in Table 2. Pre- and post-intervention SDS scores revealed a significant improvement in both the CR group $(\mathrm{P}=0.007)$ and the MTDLP group $(\mathrm{P}=0.010)$. The effect size of the SDS scores was also large in both the CR group $(r=0.72)$ and the MTDLP group ( $\mathrm{r}=0.78)$. The FAI scores of patients in the MTDLP group showed a significant improvement $(\mathrm{P}=0.003)$. Furthermore, the effect sizes of the FAI $(\mathrm{r}=0.89)$ and LSA $(r=0.52)$ scores in the MTDLP group were also large. Moreover, two-way analysis of variance indicated that the SDS scores of the CR and MTDLP groups had a significant main effect for the period ( $\mathrm{P}=0.000)$. However, no significant differences were noted in any of the items related to the main effect of the group or related to their interaction.

\section{DISCUSSION}

\section{Effectiveness of Intervention Using MTDLP}

To the best of our knowledge, this is the first study to have evaluated the effectiveness of intervention using MTDLP on patients with cardiovascular diseases. Comparing the CR and MTDLP groups, there were no significant differences in exercise tolerance functions or heart disease severity. The subjects of this study were in a depressed state, but their heart function was stable and they were able to live independently at home. Comparisons of parameter values before and after intervention in the CR and MTDLP groups indicated that CR improved depression and that intervention using MTDLP along with CR improved not only depression but also APDL.

Bita et al. ${ }^{27)}$ reported that the FAI average was $22.05 \pm 8.33$ in an FAI-based survey of 66 people over 65 years of age in a Canadian-living Chinese community. In addition, Soham et al. ${ }^{28)}$ reported that the LSA average was $41.7 \pm 20.9$ in a survey using LSA for elderly people over 75 years of age in Mexico. Other investigations ${ }^{25,26)}$ have also reported that the 
Table 1. Basic characteristics of patients

\begin{tabular}{|c|c|c|c|}
\hline & CR group $(n=14)$ & MTDLP group $(n=11)$ & $\mathrm{P}$ \\
\hline Sex: M/F & $9 / 5$ & $7 / 4$ & 0.648 \\
\hline Age (years) & $75.6 \pm 4.1$ & $73.2 \pm 9.3$ & 1.00 \\
\hline $\operatorname{BMI}\left(\mathrm{kg} / \mathrm{m}^{2}\right)$ & $27.1 \pm 4.8$ & $23.7 \pm 3.7$ & 0.070 \\
\hline Primary disease & & & 0.070 \\
\hline Angina pectoris & 10 & 3 & \\
\hline Myocardial infarction & 4 & 3 & \\
\hline Chronic heart failure & 0 & 2 & \\
\hline After open surgery & 0 & 2 & \\
\hline Cardiovascular disease & 0 & 1 & \\
\hline \multicolumn{4}{|l|}{ Cardiac function } \\
\hline LVEF (\%) & $66.3 \pm 6.3$ & $56.0 \pm 15.9$ & 0.082 \\
\hline $\mathrm{E} / \mathrm{e}^{\prime}$ & $12.4 \pm 2.5$ & $15.7 \pm 11.1$ & 0.853 \\
\hline NYHA classification of cardiac performance & & & 0.158 \\
\hline I & 11 & 11 & \\
\hline II & 3 & 0 & \\
\hline III & 0 & 0 & \\
\hline IV & 0 & 0 & \\
\hline \multicolumn{4}{|l|}{ Renal function } \\
\hline eGFR (ml/min/1.73 m²) & $52.6 \pm 12.8$ & $48.9 \pm 10.9$ & 0.474 \\
\hline \multicolumn{4}{|l|}{ Exercise tolerance functions } \\
\hline Grip strength (kg) & $24.8 \pm 9.9$ & $26.5 \pm 9.0$ & 0.519 \\
\hline Knee extension strength (kg) & $25.8 \pm 11.7$ & $27.8 \pm 10.7$ & 0.516 \\
\hline TUG (s) & $7.5 \pm 2.0$ & $7.7 \pm 3.0$ & 0.849 \\
\hline
\end{tabular}

Values are mean $\pm \mathrm{SD}$ or $\mathrm{n}$ or as otherwise indicated.

Table 2. Outcome measures before and after intervention

\begin{tabular}{|c|c|c|c|c|c|c|c|c|c|c|c|}
\hline & \multicolumn{4}{|c|}{ CR group $(n=14)$} & \multicolumn{4}{|c|}{ MTDLP group $(n=11)$} & \multicolumn{3}{|c|}{ Two-way ANOVA } \\
\hline & Pre- & & & & $\mathrm{Dm}$ & & & & Main & effect & Interaction \\
\hline & intervention & intervention & 1 & t & intervention & intervention & 1 & & Group (P) & Period $(\mathrm{P})$ & Group $\times$ Period \\
\hline SDS & $52.9 \pm 3.9$ & $48.9 \pm 6.6$ & $0.007 * *$ & 0.72 & $53.0 \pm 4.4$ & $44.4 \pm 7.3$ & $0.010^{*}$ & 0.78 & 0.187 & $0.000 * *$ & 0.160 \\
\hline BI & $98.9 \pm 2.9$ & $99.3 \pm 2.7$ & 0.785 & 0.07 & $99.6 \pm 1.5$ & $99.6 \pm 1.5$ & 1.000 & 0.00 & 0.510 & 0.788 & 0.788 \\
\hline FAI & $17.4 \pm 9.0$ & $19.2 \pm 9.8$ & 0.069 & 0.49 & $15.8 \pm 8.4$ & $23.3 \pm 6.8$ & $0.003 * *$ & 0.89 & 0.622 & 0.068 & 0.257 \\
\hline LSA & $79.5 \pm 26.5$ & $82.1 \pm 23.2$ & 0.937 & 0.02 & $73.9 \pm 18.2$ & $86.7 \pm 18.9$ & 0.083 & 0.52 & 0.382 & 0.509 & 0.319 \\
\hline
\end{tabular}

Values are mean \pm SD or as otherwise indicated.

ANOVA, analysis of variance.

$* \mathrm{P}<0.05 * * \mathrm{P}<0.01$.

average LSA was between 53.3 and 64.1. In this study, the FAI averages of the CR and MTDLP groups were 17.4 \pm 9.0 and $15.8 \pm 8.4$, respectively. Furthermore, the LSA averages of the CR and MTDLP groups were $79.5 \pm 26.5$ and 73.9 \pm 18.2 , respectively. That is, the subjects of this study were ADL self-sustaining and had a wide life space, but they were in a depressed state and had declining APDL.
In the current study, during the MTDLP interviews, the OTs asked each subject about the life activities they wanted to improve. This allowed the OTs to set goals for each individual according to their life activities, such as walking for leisure and using public transportation instead of specialist taxis. Because the subjects of this study were ADL selfsustaining, their goals were related to APDL. Therefore, it is 
believed that their FAI improved significantly.

The JAOT indicates that MTDLP is useful at any stage in each field. ${ }^{29)}$ Although the results of our study differ in terms of disease, they are consistent with the results of a study conducted by the JAOT that investigated the efficacy of MTDLP. ${ }^{12)}$ This JAOT study reported an improvement in FAI items associated with housework, going outdoors, and leisure time as a result of intervention using MTDLP for patients receiving assistance with housework. The intervention using MTDLP in outpatient CR suggested that it is possible to tailor the approach method to individuals, and it is a means of effective intervention for psychological aspects such as depression.

Furthermore, interventions using MTDLP are recognized as improving physical and social activities. Inomata et al. ${ }^{30)}$ reported that interventions using MTDLP with 21 elderly people at a geriatric health services facility helped 15 of them to return to their homes and improved their ADL. In addition, Namasu ${ }^{31)}$ reported that intervention using MTDLP for patients with chronic heart failure restored physical and social activities and allowed them to continue horticultural work like before.

Because our results also suggested that intervention using MTDLP in outpatient CR improves not only depression but also the level of physical and social activity, including APDL, we expect that the involvement of OTs in CR will contribute to improvements in the prognoses of patients with cardiovascular diseases.

\section{Limitations}

Our subjects were patients undergoing outpatient CR; however, the analytical sample size was smaller than the original subject population. In the present study, intervention using MTDLP was determined by single-blind sampling; however, because of the unique characteristics of the intervention using MTDLP in this study, it was difficult to implement double-blind sampling. Furthermore, because no interaction was observed in this study, it was considered that comparisons based on parameter values before and after intervention were acceptable. Although the average FAI score in men is known to be lower than that in women, we did not discuss the improvement in FAI items based on gender differences.

\section{CONCLUSIONS}

This study examined the effectiveness of intervention using MTDLP for patients with cardiovascular diseases. Our results indicated that $\mathrm{CR}$ improves depression and that additional intervention using MTDLP improves not only depression but also APDL. Intervention using MTDLP in CR was found to be effective for psychological aspects, such as depression. Studies involving a larger sample size are warranted in the future.

\section{ACKNOWLEDGMENTS}

The authors would like to express their appreciation to all the participants in this study as well as to the staff members of the Nara Prefectural Seiwa Medical Center.

\section{CONFLICT OF INTEREST}

The authors declare no conflict of interest or financial benefit connected with this study.

\section{REFERENCES}

1. Rozanski A, Blumenthal JA, Kaplan J: Impact of psychological factors on the pathogenesis of cardiovascular disease and implications for therapy. Circulation 1999;99:2192-2217. PMID:10217662, DOI:10.1161/01. CIR.99.16.2192

2. Rutledge T, Reis VA, Linke SE, Greenberg BH, Mills PJ: Depression in heart failure: a meta-analytic review of prevalence, intervention effects, and associations with clinical outcomes. J Am Coll Cardiol 2006;48:1527-1537. PMID:17045884, DOI:10.1016/j. jacc.2006.06.055

3. Sherwood A, Blumenthal JA, Trivedi R, Johnson KS, O’Connor CM, Adams KF Jr, Dupree CS, Waugh RA, Bensimhon DR, Gaulden L, Christenson RH, Koch GG, Hinderliter AL: Relationship of depression to death or hospitalization in patients with heart failure. Arch Intern Med 2007;167:367-373. PMID:17325298, DOI:10.1001/archinte.167.4.367

4. Kawakami N, Takeshima T, Ono Y, Uda H, Hata Y, Nakane Y, Nakane H, Iwata N, Furukawa TA, Kikkawa T: Twelve-month prevalence, severity, and treatment of common mental disorders in communities in Japan: preliminary finding from the World Mental Health Japan Survey 2002-2003. Psychiatry Clin Neurosci 2005;59:441-452. PMID:16048450, DOI:10.1111/j.1440-1819.2005.01397.x 
5. The Japanese Circulation Society: Guidelines for the Diagnosis and Treatment of Circulatory Diseases (Report of the FY2011 Joint Research Team). Osaka, Available from: http://www.jacr.jp/web/pdf/JCS2012 nohara_d_2015.01.14.pdf [cited 2018 May 27].

6. Gehi A, Haas D, Pipkin S, Whooley MA: Depression and medication adherence in outpatients with coronary heart disease: findings from the Heart and Soul Study. Arch Intern Med 2005;165:2508-2513. PMID:16314548, DOI:10.1001/archinte.165.21.2508

7. Clark AM, Haykowsky M, Kryworuchko J, MacClure T, Scott J, DesMeules M, Luo W, Liang Y, McAlister FA: A meta-analysis of randomized control trials of home-based secondary prevention programs for coronary artery disease. Eur J Cardiovasc Prev Rehabil 2010;17:261-270. PMID:20560165, DOI:10.1097/ HJR.0b013e32833090ef

8. Thombs BD, de Jonge P, Coyne JC, Whooley MA, Frasure-Smith N, Mitchell AJ, Zuidersma M, EzeNliam C, Lima BB, Smith CG, Soderlund K, Ziegelstein RC: Depression screening and patient outcomes in cardiovascular care: a systematic review. JAMA 2008;300:2161-2171. PMID:19001627, DOI:10.1001/ jama.2008.667

9. Daley A: Exercise and depression: a review of reviews. J Clin Psychol Med Settings 2008;15:140-147. PMID:19104978, DOI:10.1007/s10880-008-9105-z

10. Blumenthal JA, Babyak MA, O’Connor C, Keteyian S, Landzberg J, Howlett J, Kraus W, Gottlieb S, Blackburn G, Swank A, Whellan DJ: Effects of exercise training on depressive symptoms in patients with chronic heart failure: the HF-ACTION randomized trial. JAMA 2012;308:465-474. PMID:22851113, DOI:10.1001/ jama.2012.8720

11. Frasure-Smith N, Lespérance F: Depression and other psychological risks following myocardial infarction. Arch Gen Psychiatry 2003;60:627-636. PMID:12796226, DOI:10.1001/archpsyc.60.6.627

12. The Japanese Association of Occupational Therapists: FY2009 Report of the Program to Maintain and Improve the Health of the Elderly "Comprehensive Service Model Survey Study using Comprehensive Management of Independence Support”. Osaka, Available from: http://www.jaot.or.jp/science/h21kenkyujigyo. html [cited 2018 Jun 25].
13. The Japanese Association of Occupational Therapists: FY2010 Report of Program to Maintain and Improve the Health of the Elderly "Research Program on the Vision for the Comprehensive Service Model that Utilizes Comprehensive Management". Osaka, Available from: http://www.jaot.or.jp/science/h22rokenjigyohoukokusyo.html [cited 2018 Jun 25].

14. Duruturk N, Tonga E, Karatas M, Doganozu E: Activity performance problems of patients with cardiac diseases and their impact on quality of life. J Phys Ther Sci 2015;27:2023-2028. PMID:26311919, DOI:10.1589/ jpts. 27.2023

15. The Japanese Association of Occupational Therapists: Process of the Management Tool for Daily Life Performance. Nara, Available from: http://www.jaot.or.jp/ wp-content/uploads/2014/12/managementsheet.docx [cited 2018 May 12].

16. Fukuda K, Kobayashi S: Japanese self-rating depression scale user guidance augmented version [in Japanese]. Sankyobo, Kyoto, 2011;3-6.

17. Okimoto JT, Barnes RF, Veith RC, Raskind MA, Inui TS, Carter WB: Screening for depression in geriatric medical patients. Am J Psychiatry 1982;139:799-802. PMID:7081496, DOI:10.1176/ajp.139.6.799

18. Niino N: The reliability and validity of a self-assessment type depression scale for the elderly [in Japanese]. Jpn Soc Public Health 1988;35:201-203.

19. Mahoney FI, Barthel DW: Functional evaluation: the Barthel Index. Md State Med J 1965;14:61-65. PMID:14258950

20. Suenage H, Miyanaga K, Chisaka H, Kawatsu R, Hachisuka K: The reproducibility and validity of the Revised Frenchay Activities Index Self-assessment Table [in Japanese]. J Occup Med 2000;48:55-60.

21. Holbrook M, Skilbeck C: An activities index for use with stroke patients. Age Ageing 1983;12:166-170. PMID:6869117, DOI:10.1093/ageing/12.2.166

22. Sainsbury A, Seebass G, Bansal A, Young JB: Reliability of the Barthel Index when used with older people. Age Ageing 2005;34:228-232. PMID:15863408, DOI:10.1093/ageing/afi063

23. Jones DM, Song X, Rockwood K: Operationalizing a frailty index from a standardized comprehensive geriatric assessment. J Am Geriatr Soc 2004;52:19291933. PMID:15507074, DOI:10.1111/j.15325415.2004.52521.x 
24. Hachisuka K, Chisaka H, Kawazu T, Saeki S, Negayama S: Standard values for the Frenchay Activities Index for middle-aged and elderly people classified based on random sampling of daily behaviors [in Japanese]. Jpn J Rehabil Med 2001;38:287-295. DOI:10.2490/ jjrm1963.38.287

25. Baker PS, Bodner EV, Allman RM: Measuring lifespace mobility in community-dwelling older adults. J Am Geriatr Soc 2003;51:1610-1614. PMID:14687391, DOI:10.1046/j.1532-5415.2003.51512.x

26. Peel C, Sawyer Baker P, Roth DL, Brown CJ, Brodner EV, Allman RM: Assessing mobility in older adults: the UAB Study of Aging Life-Space Assessment. Phys Ther 2005;85:1008-1119. PMID:16180950

27. Imam B, Miller WC: Reliability and validity of scores of a Chinese version of the Frenchay Activities Index. Arch Phys Med Rehabil 2012;93:520-526. PMID:22209474, DOI:10.1016/j.apmr.2011.07.197
28. Snih SA, Peek KM, Sawyer P, Markides KS, Allman $\mathrm{RM}$, Ottenbacher KJ: Life-space mobility in Mexican Americans aged 75 and older. J Am Geriatr Soc 2012;60:532-537. PMID:22283683, DOI:10.1111/j.15325415.2011.03822.x

29. The Japanese Association of Occupational Therapists: Management of Daily Life Performance based on learning derived from cases [in Japanese]. 1st ed. Ishiyaku Publishing, Inc., Tokyo, 2015; p47.

30. Inomata E, Miura A, Ishii T, Miyauchi J, Watanabe M, Kobayashi K: Early intervention using the management tool for daily life performance in the return home support of a geriatric health services facility. JOTR 2017;36:97-104.

31. Namasu Y: A case of aiming to resume farm work at home garden after discharge. JJOT 2016;50:821-825. 